\title{
Bounded Nonlinear Functional Derived by the Generalized Srivastava-Owa Fractional Differential Operator
}

\author{
Rabha W. Ibrahim \\ Institute of Mathematical Sciences, University of Malaya, 50603 Kuala Lumpur, Malaysia \\ Correspondence should be addressed to Rabha W. Ibrahim; rabhaibrahim@yahoo.com
}

Received 25 November 2012; Revised 6 January 2013; Accepted 6 January 2013

Academic Editor: Alain Miranville

Copyright (c) 2013 Rabha W. Ibrahim. This is an open access article distributed under the Creative Commons Attribution License, which permits unrestricted use, distribution, and reproduction in any medium, provided the original work is properly cited.

By making use of the generalized Srivastava-Owa fractional differential operator, a class of analytical functions is imposed. The sharp bound for the nonlinear functional associated with the Hankel determinant is computed. We consider a new technique to prove our results. Important properties such as inclusion, subordination, and Hadamard product are studied. Some recent results are included.

\section{Introduction}

Fractional calculus (real and complex) is a rapidly growing subject of interest for physicists and mathematicians. The reason for this is that problems may be discussed in a much more stringent and elegant way than using traditional methods. Fractional differential equations have emerged as a new branch of applied mathematics which has been used for many mathematical models in science and engineering. In fact, fractional differential equations are considered as an alternative model to nonlinear differential equations. Several different derivatives were introduced: Riemann-Liouville, Hadamard, Grunwald-Letnikov, Riesz, Erdelyi-Kober operators, and Caputo [1-7].

Recently, the theory of fractional calculus has found interesting applications in the theory of analytic functions. The classical definitions of fractional operators and their generalizations have fruitfully been employed for imposing, for example, the characterization properties, coefficient estimates [8], distortion inequalities [9], and convolution structures for various subclasses of analytic functions and the works in the research monographs. In [10], Srivastava and Owa defined the fractional operators (derivative and integral) in the complex $z$-plane $\mathbb{C}$ as follows.
Definition 1. The fractional derivative of order $\alpha$ is defined, for a function $f(z)$ by

$$
D_{z}^{\alpha} f(z):=\frac{1}{\Gamma(1-\alpha)} \frac{d}{d z} \int_{0}^{z} \frac{f(\zeta)}{(z-\zeta)^{\alpha}} d \zeta ; \quad 0 \leq \alpha<1
$$

where the function $f(z)$ is analytical in simply-connected region of the complex $z$-plane $\mathbb{C}$ containing the origin and the multiplicity of $(z-\zeta)^{-\alpha}$ is removed by requiring $\log (z-\zeta)$ to be real when $(z-\zeta)>0$.

Definition 2. The fractional integral of order $\alpha$ is defined, for a function $f(z)$, by

$$
I_{z}^{\alpha} f(z):=\frac{1}{\Gamma(\alpha)} \int_{0}^{z} f(\zeta)(z-\zeta)^{\alpha-1} d \zeta ; \quad \alpha>0
$$

where the function $f(z)$ is analytical in simply connected region of the complex $z$-plane $(\mathbb{C})$ containing the origin and the multiplicity of $(z-\zeta)^{\alpha-1}$ is removed by requiring $\log (z-\zeta)$ to be real when $(z-\zeta)>0$. 
In [11], the author generalized a formula for the fractional integral as follows: for natural $n \in \mathbb{N}=\{1,2, \ldots\}$ and real $\mu$, the $n$-fold integral of the form

$$
I_{z}^{\alpha, \mu} f(z)=\int_{0}^{z} \zeta_{1}^{\mu} d \zeta_{1} \int_{0}^{\zeta_{1}} \zeta_{2}^{\mu} d \zeta_{2} \cdots \int_{0}^{\zeta_{n-1}} \zeta_{n}^{\mu} f\left(\zeta_{n}\right) d \zeta_{n}
$$

Employing the Dirichlet technique implies

$$
\begin{aligned}
\int_{0}^{z} \zeta_{1}^{\mu} d \zeta_{1} \int_{0}^{\zeta_{1}} \zeta^{\mu} f(\zeta) d \zeta & =\int_{0}^{z} \zeta^{\mu} f(\zeta) d \zeta \int_{\zeta}^{z} \zeta_{1}^{\mu} d \zeta_{1} \\
& =\frac{1}{\mu+1} \int_{0}^{z}\left(z^{\mu+1}-\zeta^{\mu+1}\right) \zeta^{\mu} f(\zeta) d \zeta
\end{aligned}
$$

Repeating the above step $n-1$ times yields

$$
\begin{aligned}
& \int_{0}^{z} \zeta_{1}^{\mu} d \zeta_{1} \int_{0}^{\zeta_{1}} \zeta_{2}^{\mu} d \zeta_{2} \cdots \int_{0}^{\zeta_{n-1}} \zeta_{n}^{\mu} f\left(\zeta_{n}\right) d \zeta_{n} \\
& =\frac{(\mu+1)^{1-n}}{(n-1) !} \int_{0}^{z}\left(z^{\mu+1}-\zeta^{\mu+1}\right)^{n-1} \zeta^{\mu} f(\zeta) d \zeta
\end{aligned}
$$

which imposes the fractional operator type

$$
I_{z}^{\alpha, \mu} f(z)=\frac{(\mu+1)^{1-\alpha}}{\Gamma(\alpha)} \int_{0}^{z}\left(z^{\mu+1}-\zeta^{\mu+1}\right)^{\alpha-1} \zeta^{\mu} f(\zeta) d \zeta
$$

where $\alpha$ and $\mu \neq-1$ are real numbers and the function $f(z)$ is analytic in simply connected region of the complex $z$-plane $\mathbb{C}$ containing the origin and the multiplicity of $\left(z^{\mu+1}-\zeta^{\mu+1}\right)^{-\alpha}$ is removed by requiring $\log \left(z^{\mu+1}-\zeta^{\mu+1}\right)$ to be real when $\left(z^{\mu+1}-\right.$ $\left.\zeta^{\mu+1}\right)>0$. When $\mu=0$, we arrive at the standard SrivastavaOwa fractional integral. Further information can be found in [11].

Corresponding to the fractional integral operator, the fractional differential operator is

$$
\begin{array}{r}
D_{z}^{\alpha, \mu} f(z):=\frac{(\mu+1)^{\alpha}}{\Gamma(1-\alpha)} \frac{d}{d z} \int_{0}^{z} \frac{\zeta^{\mu} f(\zeta)}{\left(z^{\mu+1}-\zeta^{\mu+1}\right)^{\alpha}} d \zeta ; \\
0 \leq \alpha<1,
\end{array}
$$

where the function $f(z)$ is analytical in simply connected region of the complex $z$-plane $\mathbb{C}$ containing the origin and the multiplicity of $\left(z^{\mu+1}-\zeta^{\mu+1}\right)^{-\alpha}$ is removed by requiring $\log \left(z^{\mu+1}-\zeta^{\mu+1}\right)$ to be real when $\left(z^{\mu+1}-\zeta^{\mu+1}\right)>0$. We have

$$
D_{z}^{\alpha, \mu} z^{\nu}=\frac{(\mu+1)^{\alpha-1} \Gamma((\nu /(\mu+1))+1)}{\Gamma((\nu /(\mu+1))+1-\alpha)} z^{(1-\alpha)(\mu+1)+v-1} .
$$

Let $\mathscr{A}$ denote the class of functions $f(z)$ normalized by

$$
f(z)=z+\sum_{n=2}^{\infty} a_{n} z^{n}, \quad z \in U
$$

Also, let $\mathcal{S}, \mathcal{S}^{*}$ and $\mathscr{C}$ denote the subclasses of $\mathscr{A}$ consisting of functions which are, respectively, univalent, starlike $\mathbb{R}\left(z f^{\prime}(z) / f(z)\right)>0$, and convex $\mathbb{R}\left(1+\left(z^{2} f^{\prime \prime}(z) / f^{\prime}(z)\right)\right)>0$ in $U$. It is well known that, if the function $f(z)$ given by (9) is in the class $\mathcal{S}$, then $\left|a_{n}\right| \leq n, n \in \mathbb{N} \backslash\{1\}$. Moreover, if the function $f(z)$ given by (9) is in the class $\mathscr{C}$, then $\left|a_{n}\right| \leq 1, n \in$ $\mathbb{N}$.

In our present investigation, we will also make use of the Fox-Wright generalization $\Psi_{p}[z]$ of the hypergeometric ${ }_{q} F_{p}$ function defined by [12]

$$
\begin{aligned}
q_{p}^{\Psi}\left[\begin{array}{l}
\left(\alpha_{1}, A_{1}\right), \ldots,\left(\alpha_{q}, A_{q}\right) ; \\
\left(\beta_{1}, B_{1}\right), \ldots,\left(\beta_{p}, B_{p}\right) ;
\end{array}\right] \\
\quad={ }_{q} \Psi_{p}\left[\left(\alpha_{j}, A_{j}\right)_{1, q} ;\left(\beta_{j}, B_{j}\right)_{1, p} ; z\right] \\
:=\sum_{n=0}^{\infty}=\frac{\Gamma\left(\alpha_{1}+n A_{1}\right) \cdots \Gamma\left(\alpha_{q}+n A_{q}\right)}{\Gamma\left(\beta_{1}+n B_{1}\right) \cdots \Gamma\left(\beta_{p}+n B_{p}\right)} \frac{z^{n}}{n !} \\
=\sum_{n=0}^{\infty} \frac{\prod_{j=1}^{q} \Gamma\left(\alpha_{j}+n A_{j}\right)}{\prod_{j=1}^{q} \Gamma\left(\beta_{j}+n B_{j}\right)} \frac{z^{n}}{n !},
\end{aligned}
$$

where $A_{j}>0$ for all $j=1, \ldots, q, B_{j}>0$ for all $j=1, \ldots, p$, and $1+\sum_{j=1}^{p} B_{j}-\sum_{j=1}^{q} A_{j} \geq 0$ for suitable values $|z|<1$, and $\alpha_{i}, \beta_{j}$ are complex parameters.

It is well known that

$$
\begin{aligned}
q_{p}^{\Psi}\left[\begin{array}{l}
\left(\alpha_{1}, 1\right), \ldots,\left(\alpha_{q}, 1\right) ; \\
\left(\beta_{1}, 1\right), \ldots,\left(\beta_{p}, 1\right) ;
\end{array}\right] \\
\quad=\Lambda_{q}^{-1} F_{p}\left(\alpha_{1}, \ldots, \alpha_{q}, \beta_{1}, \ldots, \beta_{p} ; z\right),
\end{aligned}
$$

where

$$
\Lambda:=\frac{\prod_{j=1}^{p} \Gamma\left(\beta_{j}\right)}{\prod_{i=1}^{q} \Gamma\left(\alpha_{i}\right)}
$$

and ${ }_{q} F_{p}$ is the generalized hypergeometric function.

Now by making use of the operator (7), we introduce the following extension operator $\Phi^{\alpha, \mu}: \mathscr{A} \rightarrow \mathscr{A}$ :

$$
\begin{aligned}
\Phi^{\alpha, \mu} f(z):= & \frac{\Gamma((1 /(\mu+1))+1-\alpha)}{(\mu+1)^{\alpha-1} \Gamma((1 /(\mu+1))+1)} \\
& \times z^{\alpha-\mu+\mu \alpha} D_{z}^{\alpha, \mu} f(z) \\
= & \frac{\Gamma((1 /(\mu+1))+1-\alpha)}{(\mu+1)^{\alpha-1} \Gamma((1 /(\mu+1))+1)} z^{\alpha-\mu+\mu \alpha} D_{z}^{\alpha, \mu} \\
& \times\left(z+\sum_{n=2}^{\infty} a_{n} z^{n}\right) \\
= & \frac{\Gamma((1 /(\mu+1))+1-\alpha)}{(\mu+1)^{\alpha-1} \Gamma((1 /(\mu+1))+1)} z^{\alpha-\mu+\mu \alpha}
\end{aligned}
$$




$$
\begin{aligned}
\times & {\left[\frac{(\mu+1)^{\alpha-1} \Gamma((1 /(\mu+1))+1)}{\Gamma((1 /(\mu+1))+1-\alpha)} z^{-\alpha+\mu-\mu \alpha+1}\right.} \\
& \left.+\sum_{n=2}^{\infty} \frac{\Gamma((n /(\mu+1))+1)}{\Gamma((n /(\mu+1))+1-\alpha)} a_{n} z^{n-\alpha+\mu-\mu \alpha}\right] \\
= & +\sum_{n=2}^{\infty} \frac{\Gamma((1 /(\mu+1))+1-\alpha)}{\Gamma((1 /(\mu+1))+1)} \\
& \times \frac{\Gamma((n /(\mu+1))+1)}{\Gamma((n /(\mu+1))+1-\alpha)} a_{n} z^{n} \\
:= & z+\sum_{n=2}^{\infty} \phi_{n}^{\alpha, \mu} a_{n} z^{n} .
\end{aligned}
$$

Obviously, when $\mu=0$, we have the extension fractional differential operator defined in [13] ([14] for recent work), which contains the Carlson-Shaffer operator. In term of the Fox-Wright generalized function,

$$
\begin{aligned}
\Phi^{\alpha, \mu} f(z)= & z \sum_{n=2}^{\infty} \frac{\Gamma(n+1) \Gamma((1 /(\mu+1))+1-\alpha)}{\Gamma((1 /(\mu+1))+1)} \\
& \times \frac{\Gamma((n /(\mu+1))+1)}{\Gamma((n /(\mu+1))+1-\alpha)} \frac{a_{n}}{n !} z^{n} \\
= & \sum_{n=0}^{\infty} \frac{\Gamma(n+1) \Gamma((1 /(\mu+1))+1-\alpha)}{\Gamma((1 /(\mu+1))+1)} \\
& \times \frac{\Gamma((n /(\mu+1))+1)}{\Gamma((n /(\mu+1))+1-\alpha)} \frac{a_{n}}{n !} z^{n} \\
= & \frac{\Gamma((1 /(\mu+1))+1-\alpha)}{\Gamma((1 /(\mu+1))+1)} \\
& \times{ }_{2} \Psi_{1}\left[\begin{array}{c}
(1,1),\left(1, \frac{1}{\mu+1}\right) ; \\
\mu+1
\end{array}\right) ; \\
= & \frac{\Gamma((1 /(\mu+1))+1-\alpha)}{\Gamma((1 /(\mu+1))+1)}{ }_{2} \Psi_{1}[z] * f(z) \\
:= & \Psi(\alpha, \mu ; z) * f(z), \\
&
\end{aligned}
$$

where $a_{0}=0, a_{1}=1$, and $*$ is the Hadamard product. Note that

$$
\begin{gathered}
\Phi^{0,0} f(z)=f(z), \quad \Phi^{1,0} f(z)=z f^{\prime}(z), \\
\left(\Phi^{\alpha, 0} f\right)(z)=(L(2,2-\alpha) f)(z),
\end{gathered}
$$

where $L(a, c) f$ is the Carlson-Shaffer operator. Moreover, operator (14) can be viewed as a linear operator which is essentially analogous to the Dziok-Srivastava operator whenever used instead of the Fox-Wright generalization of the hypergeometric function.
Recently, various results, such as convolution and inclusion properties, distortion theorem, extreme points, and coefficient estimates, are proposed by many authors for the operators due to Srivastava involving the Wright function, generalized hypergeometric function, and Meijer's $G$-functions. These operators are Dziok-Srivastava, Srivastava-Wright, Cho-Kwon-Srivastava operator, Cho-Saigo-Srivastava operator, Jung-Kim-Srivastava, and Srivastava-Owa operators (see [15-24]). Going on in this generalization, we have finally the Erdelyi-Kober operator of fractional integration with three parameters used in [25].

Definition 3 (subordination principal). For two functions $f$ and $g$ analytical in $U$, we say that the function $f(z)$ is subordinated to $g(z)$ in $U$ and write $f(z) \prec g(z)(z \in U)$, if there exists a Schwartz function $w(z)$ analytical in $U$ with $w(0)=0$, and $|w(z)|<1$, such that $f(z)=g(w(z)), z \in U$. In particular, if the function $g(z)$ is univalent in $U$, the above subordination is equivalent to $f(0)=g(0)$ and $f(U) \subset g(U)$.

Definition 4. For the function $f$ defined by (9), the Hankel determinant of $f$ is defined by

$$
\left|\begin{array}{cccc}
a_{n} & a_{n+1} & \ldots & a_{n+q-1} \\
a_{n+1} & a_{n+2} & \ldots & a_{n+q} \\
\vdots & \vdots & \vdots & \vdots \\
a_{n+q-1} & a_{n+q} & \ldots & a_{n+2 q-2}
\end{array}\right|
$$

Now we proceed to define a new class of analytic function involving the operator (13).

Definition 5. The function $f \in \mathscr{A}$ is said to be in the class $\mathscr{R}_{\alpha, \mu}(\theta, \rho)$, where $0 \leq \alpha<1, \mu \geq 0,|\theta|<\pi / 2,0 \leq \rho \leq 1$, if it satisfies the inequality

$$
\mathbb{R}\left\{e^{i \theta} \frac{\Phi^{\alpha, \mu} f(z)}{z}\right\}>\rho \cos \theta, \quad(z \in U) .
$$

Consequently, from Definition 4, we have

$$
\begin{aligned}
f \in \mathscr{R}_{\alpha, \mu}(\theta, \rho) \Longleftrightarrow e^{i \theta} \frac{\Phi^{\alpha, \mu} f(z)}{z} \\
=[(1-\rho) p(z)+\rho] \cos \theta+i \sin \theta,
\end{aligned}
$$

where $p(z)=1+c_{1} z+c_{2} z^{2}+\cdots, z \in U$ satisfies the following properties [26]:

(i) $\left|c_{n}\right| \leq 2$ and $\mathbb{R}(p(z))>0$,

(ii) $2 c_{2}=c_{1}^{2}+w\left(4-c_{1}^{2}\right)$,

(iii) $4 c_{3}=c_{1}^{3}+2\left(4-c_{1}^{2}\right) c_{1} w-c_{1}\left(4-c_{1}^{2}\right) w^{2}$

$$
+2\left(4-c_{1}^{2}\right)\left(1-\left.w\right|^{2}\right) z, \quad(|w| \leq 1,|z| \leq 1) \text {. }
$$

We denote this class by $\mathscr{P}$.

Note that

$$
\mathscr{R}_{\alpha, 0}(0, \rho)=\mathbb{R}\left\{\frac{\Phi^{\alpha, 0} f(z)}{z}\right\}>\rho
$$


(see [27]),

(see [28]).

$$
\mathscr{R}_{\alpha, 0}(\theta, \rho)=\mathbb{R}\left\{e^{i \theta} \frac{\Phi^{\alpha, 0} f(z)}{z}\right\}>\rho \cos \theta
$$

It is well known that, for the univalent function $f$ of the form (9), the sharp inequality $\left|a_{3}-a_{2}^{2}\right| \leq 1$ holds. In the recent paper, we assume the Hankel determinant for $n=2, q=2$ and calculate the sharp bound for the functional $\left|a_{2} a_{4}-a_{3}^{2}\right|$ for $f \in \mathscr{R}_{\alpha, \mu}(\theta, \rho)$. Properties of this class are illustrated, and some well-known results are generalized. For this purpose, we need the following preliminary in the sequel, which can be found in [29].

Lemma 6. Let $\phi$ and $\psi$ be univalent convex in $U$. Then, the Hadamard product $\phi * \psi$ is also univalent convex function in $U$.

Lemma 7. Let $\Phi$ and $\Psi$ be univalent convex in $U$, and $\phi \prec \Phi$ and $\psi \prec \Psi$. Then, $\phi * \psi \prec \Phi * \Psi$.

Lemma 8. Let $\phi$ and $\psi$ be starlike of order $1 / 2$ then, for function $\Phi$ satisfying $\mathbb{R}(\Phi(z))>\sigma(\sigma \in[0,1))$,

$$
\mathbb{R}\left(\frac{\phi(z) * \Phi(z) \psi(z)}{\phi(z) * \psi(z)}\right)>\sigma, \quad(z \in U) .
$$

\section{Main Results}

We have the following result.

Theorem 9. Let the function $f$ be in the class $\mathscr{R}_{\alpha, \mu}(\theta, \rho)$. Then

$$
\left|a_{2} a_{4}-a_{3}^{2}\right| \leq \frac{(1-\rho)^{2} \cos ^{2} \theta}{\mu_{1}^{2} \mu_{2} \mu_{4} \mu_{3}^{2}}\left(80 \beta+16 \mu_{3}^{2}\right),
$$

where $\beta:=\left|\mu_{3}^{2}-\mu_{2} \mu_{4}\right|$ and

$$
\begin{aligned}
& \mu_{1}=\frac{\Gamma((1 /(\mu+1))+1-\alpha)}{\Gamma((1 /(\mu+1))+1)}, \\
& \mu_{2}=\frac{\Gamma((2 /(\mu+1))+1)}{\Gamma((2 /(\mu+1))+1-\alpha)}, \\
& \mu_{3}=\frac{\Gamma((3 /(\mu+1))+1)}{\Gamma((3 /(\mu+1))+1-\alpha)}, \\
& \mu_{4}=\frac{\Gamma((4 /(\mu+1))+1)}{\Gamma((4 /(\mu+1))+1-\alpha)} .
\end{aligned}
$$

The estimate (23) is sharp.

Proof. Since $f \in \mathscr{R}_{\alpha, \mu}(\theta, \rho)$, then

$$
\begin{aligned}
& e^{i \theta} \frac{\Phi^{\alpha, \mu} f(z)}{z} \\
& \quad=\left[(1-\rho)\left(1+c_{1} z+c_{2} z^{2}+\cdots\right)+\rho\right] \cos \theta+i \sin \theta
\end{aligned}
$$

Comparing the coefficients of (13) and (25), we receive

$$
\begin{gathered}
a_{2}=\frac{(1-\rho) c_{1} \cos \theta}{e^{i \theta} \mu_{1} \mu_{2}}, \quad a_{3}=\frac{(1-\rho) c_{2} \cos \theta}{e^{i \theta} \mu_{1} \mu_{3}}, \\
a_{4}=\frac{(1-\rho) c_{3} \cos \theta}{e^{i \theta} \mu_{1} \mu_{4}}
\end{gathered}
$$

where

$$
\begin{aligned}
& \mu_{1}=\frac{\Gamma((1 /(\mu+1))+1-\alpha)}{\Gamma((1 /(\mu+1))+1)}, \\
& \mu_{2}=\frac{\Gamma((2 /(\mu+1))+1)}{\Gamma((2 /(\mu+1))+1-\alpha)}, \\
& \mu_{3}=\frac{\Gamma((3 /(\mu+1))+1)}{\Gamma((3 /(\mu+1))+1-\alpha)}, \\
& \mu_{4}=\frac{\Gamma((4 /(\mu+1))+1)}{\Gamma((4 /(\mu+1))+1-\alpha)} .
\end{aligned}
$$

Therefore, (26) implies

$$
\left|a_{2} a_{4}-a_{3}^{2}\right|=\frac{(1-\rho)^{2} \cos ^{2} \theta}{\mu_{1}^{2}}\left|\frac{c_{1} c_{3}}{\mu_{2} \mu_{4}}-\frac{c_{2}^{2}}{\mu_{3}^{2}}\right| .
$$

By letting $c_{1}:=c$ and using (i)-(iii), we have

$$
\begin{aligned}
\left|a_{2} a_{4}-a_{3}^{2}\right|= & \frac{(1-\rho)^{2} \cos ^{2} \theta}{\mu_{1}^{2} \mu_{2} \mu_{4} \mu_{3}^{2}} \\
& \times \mid c^{4}\left(\mu_{3}^{2}-\mu_{2} \mu_{4}\right)+2 w c^{2}\left(4-c^{2}\right) \\
& \times\left(\mu_{3}^{2}-\mu_{2} \mu_{4}\right)-w^{2}\left(4-c^{2}\right) \\
& \times\left(\mu_{3}^{2} c^{2}+\mu_{2} \mu_{4}\left(4-c^{2}\right)\right) \\
& +\left(1-|w|^{2}\right) z\left(2 c\left(4-c^{2}\right) \mu_{3}^{2}\right) \mid .
\end{aligned}
$$

By employing the triangle inequality and assuming $|w|:=$ $x, \beta:=\left|\mu_{3}^{2}-\mu_{2} \mu_{4}\right|, c>0$, and $|z| \leq 1$, we obtain

$$
\begin{aligned}
\left|a_{2} a_{4}-a_{3}^{2}\right| \leq & \frac{(1-\rho)^{2} \cos ^{2} \theta}{\mu_{1}^{2} \mu_{2} \mu_{4} \mu_{3}^{2}} \\
& \times\left\{c^{4} \beta+2 x c^{2}\left(4-c^{2}\right) \beta\right. \\
& +x^{2}\left(4-c^{2}\right) \beta\left(c^{2}+2 c\right) \\
& \left.+2 c\left(4-c^{2}\right) \mu_{3}^{2}\right\}:=F(x, c) .
\end{aligned}
$$

Our aim is to maximize $F$ in the interior of the domain $\mathscr{D}=$ $[0,1] \times[0,2]$. Since

$$
\begin{aligned}
\frac{\partial F(x, c)}{\partial x}=\frac{(1-\rho)^{2} \cos ^{2} \theta}{\mu_{1}^{2} \mu_{2} \mu_{4} \mu_{3}^{2}}\{ & 2 c^{2}\left(4-c^{2}\right) \beta \\
& \left.+2 x\left(4-c^{2}\right) \beta\left(c^{2}+2 c\right)\right\}>0,
\end{aligned}
$$


thus $F$ cannot have a maximum in the interior of $\mathscr{D}$. Furthermore,

$$
\max _{x \in[0,1]} F(x, c)=F(1, c):=H(c),
$$

where

$$
\begin{aligned}
& H(c) \leq \frac{(1-\rho)^{2} \cos ^{2} \theta}{\mu_{1}^{2} \mu_{2} \mu_{4} \mu_{3}^{2}}[ 16 \beta+16 \beta\left(4-c^{2}\right) \\
&\left.+4\left(4-c^{2}\right) \mu_{3}^{2}\right]:=G(c) .
\end{aligned}
$$

But

$$
\max _{c \in[0,2]} G(c)=G(0)
$$

hence the upper bound of (28) is

$$
\left|a_{2} a_{4}-a_{3}^{2}\right| \leq \frac{(1-\rho)^{2} \cos ^{2} \theta}{\mu_{1}^{2} \mu_{2} \mu_{4} \mu_{3}^{2}}\left(80 \beta+16 \mu_{3}^{2}\right) .
$$

The equality holds for the functions

$$
\begin{aligned}
f(z)= & \Psi(\alpha, \mu ; z) \\
& * e^{i \theta}\left[z\left(\frac{1+(1-2 \rho) z^{2}}{1-z^{2}} \cos \theta+i \sin \theta\right)\right] .
\end{aligned}
$$

Remark 10. Letting $\mu=0$, we receive a recent result due to Mishra and Gochhayat [28]; putting $\alpha \rightarrow 1, \mu=0, \rho=0$, we obtain a result given by Janteng et al. [30].

Theorem 11. Assume that $\theta \in(-\pi / 2, \pi / 2), \quad \rho \in[0,1)$ and $\alpha_{1}, \alpha_{2} \in[0,1)$, with $\alpha_{1}<\alpha_{2}$. If the subordination

$$
\begin{aligned}
& z \mathscr{G}^{\prime \prime}\left(\frac{1}{\mu+1}+1-\alpha_{2}, \frac{1}{\mu+1}+1-\alpha_{1} ; z\right) \\
& +\mathscr{G}^{\prime}\left(\frac{1}{\mu+1}+1-\alpha_{2}, \frac{1}{\mu+1}+1-\alpha_{1} ; z\right) \prec 1+z,
\end{aligned}
$$

where

$$
\mathscr{G}(a, c ; z):=\sum_{n=0}^{\infty} \frac{(a)_{n}}{(c)_{n}} z^{n+1}, \quad(c \neq 0,-1,-2, \ldots),
$$

holds, then

$$
\mathscr{R}_{\alpha_{2}, \mu}(\theta, \rho) \subset \mathscr{R}_{\alpha_{1}, \mu}(\theta, \rho)
$$

Proof. Let $f \in \mathscr{R}_{\alpha_{2}, \mu}(\theta, \rho)$. We rewrite

$$
\begin{aligned}
\Phi^{\alpha_{1}, \mu} f(z)= & \Psi\left(\alpha_{1}, \mu ; z\right) * f(z) \\
= & \left(\Psi^{(-1)}\left(\alpha_{2}, \mu ; z\right) * \Psi\left(\alpha_{2}, \mu ; z\right)\right) \\
& * \Psi\left(\alpha_{1}, \mu ; z\right) * f(z)
\end{aligned}
$$

$$
\begin{aligned}
= & \left(\Psi^{(-1)}\left(\alpha_{2}, \mu ; z\right) * \Psi\left(\alpha_{1}, \mu ; z\right)\right) \\
& * \Psi\left(\alpha_{2}, \mu ; z\right) * f(z) \\
= & \mathscr{G}\left(\frac{1}{\mu+1}+1-\alpha_{2}, \frac{1}{\mu+1}+1-\alpha_{1} ; z\right) \\
& * \Phi^{\alpha_{2}, \mu} f(z),
\end{aligned}
$$

where

$$
\begin{aligned}
\Psi\left(\alpha_{2}, \mu ; z\right)= & \sum_{n=0}^{\infty} \frac{\Gamma(n+1) \Gamma((1 /(\mu+1))+1-\alpha)}{\Gamma((1 /(\mu+1))+1)} \\
& \times \frac{\Gamma((n /(\mu+1))+1)}{\Gamma((n /(\mu+1))+1-\alpha)} \frac{1}{n !} z^{n}, \\
\Psi^{(-1)}\left(\alpha_{2}, \mu ; z\right)= & \sum_{n=0}^{\infty} \frac{\Gamma((1 /(\mu+1))+1)}{\Gamma(n+1) \Gamma((1 /(\mu+1))+1-\alpha)} \\
& \times \frac{\Gamma((n /(\mu+1))+1-\alpha)}{\Gamma((n /(\mu+1))+1)} n ! z^{n}, \\
\mathscr{G}(a, c ; z)= & \sum_{n=0}^{\infty} \frac{(a)_{n}}{(c)_{n}} z^{n+1} \quad(c \neq 0,-1,-2, \ldots),
\end{aligned}
$$

and $(x)_{k}=\Gamma(x+k) / \Gamma(x)$ is a Pochhammer symbol. Therefore,

$$
\begin{aligned}
& \frac{e^{i \theta} \Phi^{\alpha_{1}, \mu} f(z)}{z} \\
& \quad=\frac{\mathscr{G}\left((1 /(\mu+1))+1-\alpha_{2},(1 /(\mu+1))+1-\alpha_{1} ; z\right) *\left(e^{i \theta} \Phi^{\alpha_{2}, \mu} f(z) / z\right) z}{\mathscr{G}\left((1 /(\mu+1))+1-\alpha_{2},(1 /(\mu+1))+1-\alpha_{1} ; z\right) * z} .
\end{aligned}
$$

Assumption (37) implies that $\mathscr{C}\left((1 /(\mu+1))+1-\alpha_{2},(1 /(\mu+\right.$ 1)) $\left.+1-\alpha_{1} ; z\right)$ is convex (see [31, Theorem 1.9]) and consequently $\mathscr{G}\left((1 /(\mu+1))+1-\alpha_{2},(1 /(\mu+1))+1-\alpha_{1} ; z\right) \epsilon$ $\mathcal{S}^{*}(1 / 2)$ (Marx-Strohhäcker Theorem [32]). Moreover, the function $\psi(z)=z$ is starlike of order $1 / 2$, then in view of Lemma 8, we obtain that

$$
\mathbb{R}\left(\frac{e^{i \theta} \Phi^{\alpha_{1}, \mu} f(z)}{z}\right)>\rho \cos \theta,
$$

and consequently $\mathscr{R}_{\alpha_{2}, \mu}(\theta, \rho) \subset \mathscr{R}_{\alpha_{1}, \mu}(\theta, \rho)$.

Remark 12. Condition (37) can be replaced by another condition to obtain the convexity of the function $\mathscr{G}$, such that

$$
\left|z \mathscr{G}^{\prime \prime}\left(\frac{1}{\mu+1}+1-\alpha_{2}, \frac{1}{\mu+1}+1-\alpha_{1} ; z\right)\right|<\frac{1}{2}
$$

yields that $\mathscr{G}\left((1 /(\mu+1))+1-\alpha_{2},(1 /(\mu+1))+1-\alpha_{1} ; z\right)$ is convex (see [31]).

Theorem 13. Let $f \in \mathcal{S}^{*}$ and $h \in \mathscr{R}_{\alpha, \mu}(\theta, \rho)(\rho \in[0,1], \theta \in$ $(-\pi / 2, \pi / 2), \alpha \in[0,1), \mu \geq 0)$. Then $f * h \in \mathscr{R}_{\alpha, \mu}(\theta, \rho)$. 
Proof. By employing the properties of the Hadamard product, we receive

$$
\Phi^{\alpha, \mu}(f * h)(z)=f(z) * \Phi^{\alpha, \mu} h(z) .
$$

Therefore,

$$
\frac{e^{i \theta} \Phi^{\alpha, \mu}(f * h)(z)}{z}=\frac{f(z) *\left(\left(e^{i \theta} \Phi^{\alpha, \mu} h(z)\right) / z\right) z}{f(z) * z} .
$$

In virtue of Lemma 8, we have

$$
\mathbb{R}\left(\frac{e^{i \theta} \Phi^{\alpha, \mu}(f * h)(z)}{z}\right)>\rho \cos \theta .
$$

Hence $f * h \in \mathscr{R}_{\alpha, \mu}(\theta, \rho)$.

Theorem 14. Let $f \in \mathscr{R}_{\alpha, \mu}(\theta, \rho)(\rho \in[0,1], \theta \in(-\pi / 2, \pi / 2)$, $\alpha \in[0,1), \mu \geq 0)$. Then the integral

$$
(\mathscr{I} f)(z)=\frac{\tau+1}{z^{\tau}} \int_{0}^{z} \xi^{\tau-1} f(\xi) d \xi \quad(z \in U, \tau>-1)
$$

is also in $\mathscr{R}_{\alpha, \mu}(\theta, \rho)$.

Proof. It is easy to show that

$$
\Phi^{\alpha, \mu}(\mathscr{I} f)(z)=\mathscr{G}(\tau+1, \tau+2) * \Phi^{\alpha, \mu} f(z) .
$$

Therefore,

$$
\begin{aligned}
\frac{e^{i \theta} \Phi^{\alpha, \mu}(\mathscr{I} f)(z)}{z} \\
=\frac{\mathscr{G}(\tau+1, \tau+2) *\left(\left(e^{i \theta} \Phi^{\alpha, \mu} f(z)\right) / z\right) z}{\mathscr{G}(\tau+1, \tau+2) * z} .
\end{aligned}
$$

But $\mathscr{G}(\tau+1, \tau+2) \in \mathcal{S}^{*}(1 / 2)$, thus in view of Lemma 8 , the proof is complete.

Remark 15. When $\mu=0$ in Theorems 13 and 14, we have the results given in [28].

Theorem 16. Let $\rho \in[0,1], \theta \in(-\pi / 2, \pi / 2), \alpha \in[0,1), \mu \geq$ 0 . If the subordination

$$
\begin{aligned}
\mathscr{G}^{\prime \prime} & \left(\frac{1}{\mu+1}+1-\alpha, \frac{1}{\mu+1}+1 ; z\right) \\
& -\frac{2}{z} \mathscr{G}^{\prime}\left(\frac{1}{\mu+1}+1-\alpha, \frac{1}{\mu+1}+1 ; z\right) \\
& +\frac{2}{z^{2}} \mathscr{G}\left(\frac{1}{\mu+1}+1-\alpha, \frac{1}{\mu+1}+1 ; z\right) \prec 1+z
\end{aligned}
$$

holds, then $g(z):=\left(e^{-i \theta} / z\right)(\mathscr{G}((1 /(\mu+1))+1-\alpha,(1 /(\mu+1))+$ $\left.1 ; z) * z\left(\left(\left(1+(1-2 \rho) z^{2}\right) /\left(1-z^{2}\right)\right) \cos \theta+i \sin \theta\right)\right)$ is univalent convex function.

Proof. Condition (51) yields that $\mathscr{G}(1 /(\mu+1))+1-\alpha,(1 /(\mu+$ $1))+1 ; z / z$ is univalent convex (see [31, Theorem 1.9]) and consequently, in view of Lemma $6, g(z)$ is univalent convex function.
Theorem 17. Let $f \in \mathscr{R}_{\alpha_{2}, \mu}(\theta, \rho)$. Then

$$
\frac{f(z)}{z} \prec g(z),
$$

where $g$ is defined in Theorem 16.

Proof. Since $f \in \mathscr{R}_{\alpha_{2}, \mu}(\theta, \rho)$, then we have

$$
\frac{\Phi^{\alpha, \mu} f(z)}{z} \prec e^{-i \theta}\left(\frac{1+(1-2 \rho) z^{2}}{1-z^{2}} \cos \theta+i \sin \theta\right) .
$$

But $g$ is univalent convex function (Theorem 16); thus by an application of Lemma 7, we obtain the desired assertion.

\section{Conclusion}

We defined a new fractional differential operator which generalized well-known linear and nonlinear operators such as Carlson-Shaffer operator and the Dziok-Srivastava (linear operators) and Srivastava-Owa fractional differential operators (nonlinear operator). By making uses this operator a generalized class of analytic functions is defined and studied. The sharp bound for nonlinear functional based on the second-order Hankel determinant $\left|a_{2} a_{4}-a_{3}^{2}\right|$, involving the generalized fractional differential operator, is computed. Several properties, depending on the Hadamard product, are imposed. We have shown that some results are generalized by recent works due to Mishra-Gochhayat, Ling-Ding, and Janteng et al. Furthermore, a new approach is introduced in the proof of Theorems 11 and 16 based on the subordination concept and employing the result due to Ponnusamy and Singh.

\section{References}

[1] S. G. Samko, A. A. Kilbas, and O. I. Marichev, Fractional Integrals and Derivatives: Theory and Applications, Gordon and Breach Science, Yverdon, Switzerland, 1993.

[2] I. Podlubny, Fractional Differential Equations, vol. 198, Academic Press, San Diego, Calif, USA, 1999.

[3] R. Hilfer, Applications of Fractional Calculus in Physics, World Scientific, Singapore, 2000.

[4] K. Diethelm, The Analysis of Fractional Differential Equations, vol. 2004 of Lecture Notes in Mathematics, Springer, Berlin, Germany, 2010.

[5] A. A. Kilbas, H. M. Srivastava, and J. J. Trujillo, Theory and Applications of Fractional Differential Equations, vol. 204 of North-Holland Mathematics Studies, Elsevier Science, Amsterdam, The Netherland, 2006.

[6] J. Sabatier, O. P. Agrawal, and J. A. T. Machado, Advance in Fractional Calculus: Theoretical Developments and Applications in Physics and Engineering, Springer, New York, NY, USA, 2007.

[7] V. Lakshmikantham, S. Leela, and J. V. Devi, Theory of Fractional Dynamic Systems, Cambridge Scientific, Cambridge, UK, 2009.

[8] M. Darus and R. W. Ibrahim, "Radius estimates of a subclass of univalent functions," Matematichki Vesnik, vol. 63, no. 1, pp. 55-58, 2011. 
[9] H. M. Srivastava, Y. Ling, and G. Bao, "Some distortion inequalities associated with the fractional derivatives of analytic and univalent functions," Journal of Inequalities in Pure and Applied Mathematics, vol. 2, no. 2, article 23, 6 pages, 2001.

[10] H. M. Srivastava and S. Owa, Univalent Functions, Fractional Calculus, and Their Applications, Halsted Press, John Wiley and Sons, New York, NY, USA, 1989.

[11] R. W. Ibrahim, "On generalized Srivastava-Owa fractional operators in the unit disk," Advances in Difference Equations, vol. 2011, article 55, 10 pages, 2011.

[12] H. M. Srivastava and P. W. Karlsson, Multiple Gaussian Hypergeometric Series, Halsted Press, John Wiley and Sons, New York, NY, USA, 1985.

[13] S. Owa and H. M. Srivastava, "Univalent and starlike generalized hypergeometric functions," Canadian Journal of Mathematics, vol. 39, no. 5, pp. 1057-1077, 1987.

[14] R. W. Ibrahim and M. Darus, "Differential operator generalized by fractional derivatives," Miskolc Mathematical Notes, vol. 12, no. 2, pp. 167-184, 2011.

[15] Y. Yang, Y.-Q. Tao, and J.-L. Liu, "Differential subordinations for certain meromorphically multivalent functions defined by Dziok-Srivastava operator," Abstract and Applied Analysis, vol. 2011, Article ID 726518, 9 pages, 2011.

[16] V. Kiryakova, "Criteria for univalence of the Dziok-Srivastava and the Srivastava-Wright operators in the class A," Applied Mathematics and Computation, vol. 218, no. 3, pp. 883-892, 2011.

[17] M. Darus and R. W. Ibrahim, "On the existence of univalent solutions for fractional integral equation of Volterra type in complex plane," ROMAI Journal, vol. 7, no. 1, pp. 77-86, 2011.

[18] H. M. Srivastava, M. Darus, and R. W. Ibrahim, "Classes of analytic functions with fractional powers defined by means of a certain linear operator," Integral Transforms and Special Functions, vol. 22, no. 1, pp. 17-28, 2011.

[19] K. Piejko and J. Sokól, "Subclasses of meromorphic functions associated with the Cho-Kwon-Srivastava operator," Journal of Mathematical Analysis and Applications, vol. 337, no. 2, pp. 1261-1266, 2008.

[20] J. Sokól, "On some applications of the Dziok-Srivastava operator," Applied Mathematics and Computation, vol. 201, no. 1-2, pp. 774-780, 2008.

[21] R. W. Ibrahim and M. Darus, "On analytic functions associated with the Dziok-Srivastava linear operator and Srivastava-Owa fractional integral operator," Arabian Journal for Science and Engineering, vol. 36, no. 3, pp. 441-450, 2011.

[22] B. A. Frasin, "New properties of the Jung-Kim-Srivastava integral operators," Tamkang Journal of Mathematics, vol. 42, no. 2, pp. 205-215, 2011.

[23] Z.-G. Wang, R. Aghalary, M. Darus, and R. W. Ibrahim, "Some properties of certain multivalent analytic functions involving the Cho-Kwon-Srivastava operator," Mathematical and Computer Modelling, vol. 49, no. 9-10, pp. 1969-1984, 2009.

[24] H. M. Srivastava, "Some Fox-Wright generalized hypergeometric functions and associated families of convolution operators," Applicable Analysis and Discrete Mathematics, vol. 1, no. 1, pp. 56-71, 2007, Proceedings of the International Conference on Topics in Mathematical Analysis and Graph Theory (2007).

[25] V. Kiryakova, "Convolutions of Erdélyi-Kober fractional integration operators," in Complex Analysis and Applications '87, pp. 273-283, The Bulgarian Academy of Sciences, Sofia, Bulgaria, 1989.
[26] R. J. Libera and E. J. Złotkiewicz, "Coefficient bounds for the inverse of a function with derivative in $P$," Proceedings of the American Mathematical Society, vol. 87, no. 2, pp. 251-257, 1983.

[27] L. Yi and D. Shusen, "A class of analytic functions defined by fractional derivation," Journal of Mathematical Analysis and Applications, vol. 186, no. 2, pp. 504-513, 1994.

[28] A. K. Mishra and P. Gochhayat, "Second Hankel determinant for a class of analytic functions defined by fractional derivative," International Journal of Mathematics and Mathematical Sciences, vol. 2008, Article ID 153280, 10 pages, 2008.

[29] St. Ruscheweyh and T. Sheil-Small, "Hadamard products of Schlicht functions and the Pólya-Schoenberg conjecture," Commentarii Mathematici Helvetici, vol. 48, pp. 119-135, 1973.

[30] A. Janteng, S. A. Halim, and M. Darus, "Coefficient inequality for a function whose derivative has a positive real part," Journal of Inequalities in Pure and Applied Mathematics, vol. 7, no. 2, article 50, 5 pages, 2006.

[31] S. Ponnusamy and V. Singh, "Criteria for univalent, starlike and convex functions," Bulletin of the Belgian Mathematical Society, vol. 9, no. 4, pp. 511-531, 2002.

[32] S. S. Miller and P. T. Mocanu, Differential Subordinantions: Theory and Applications, vol. 225 of Pure and Applied Mathematics, Dekker, New York, NY, USA, 2000. 


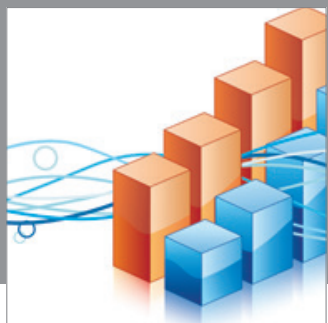

Advances in

Operations Research

mansans

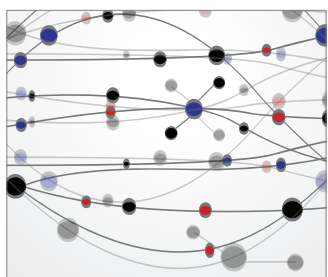

The Scientific World Journal
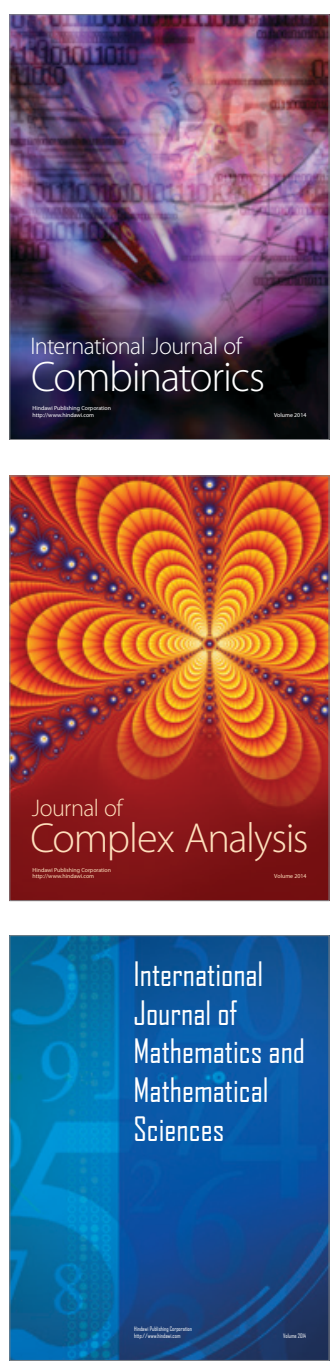
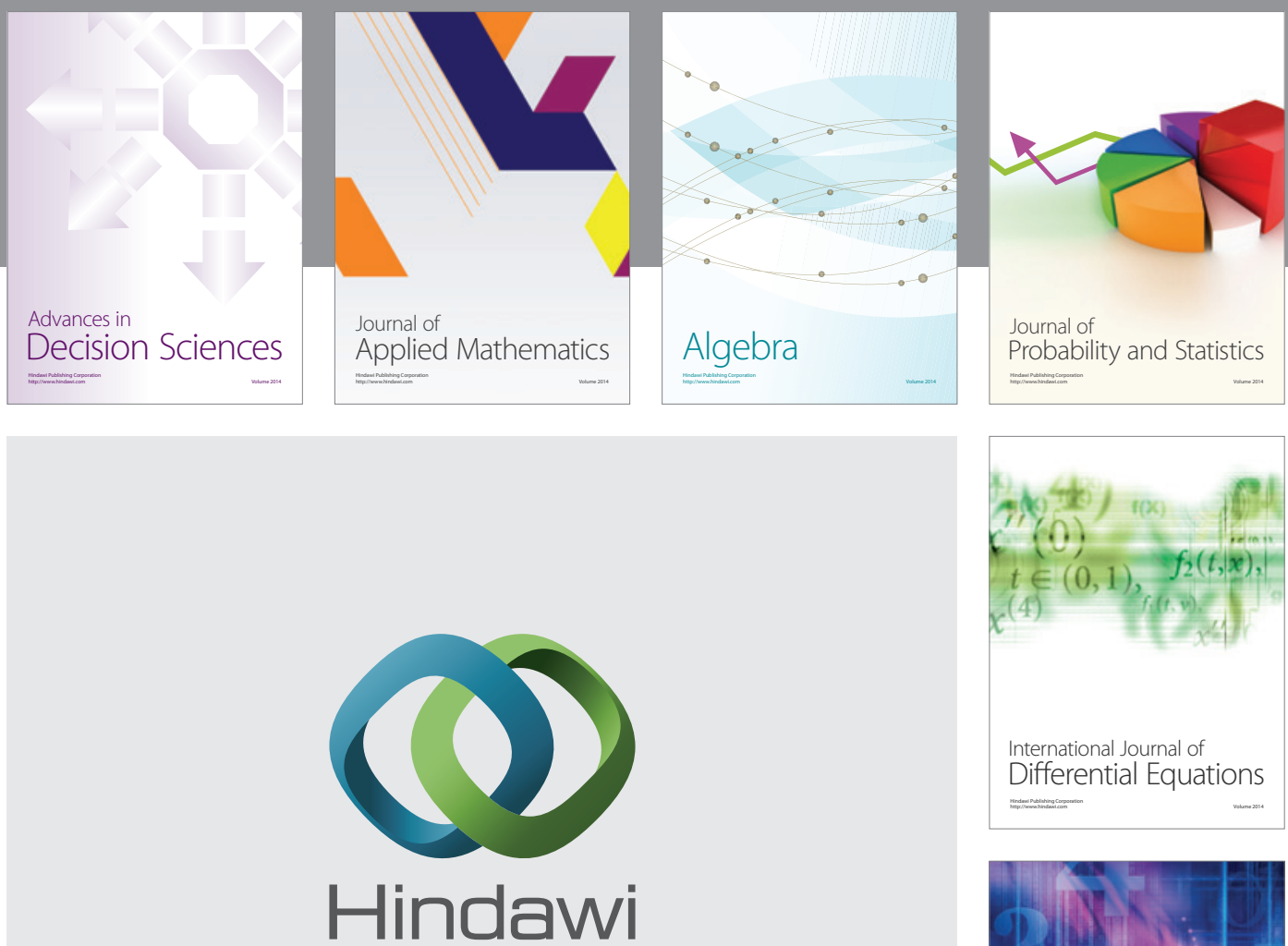

Submit your manuscripts at http://www.hindawi.com
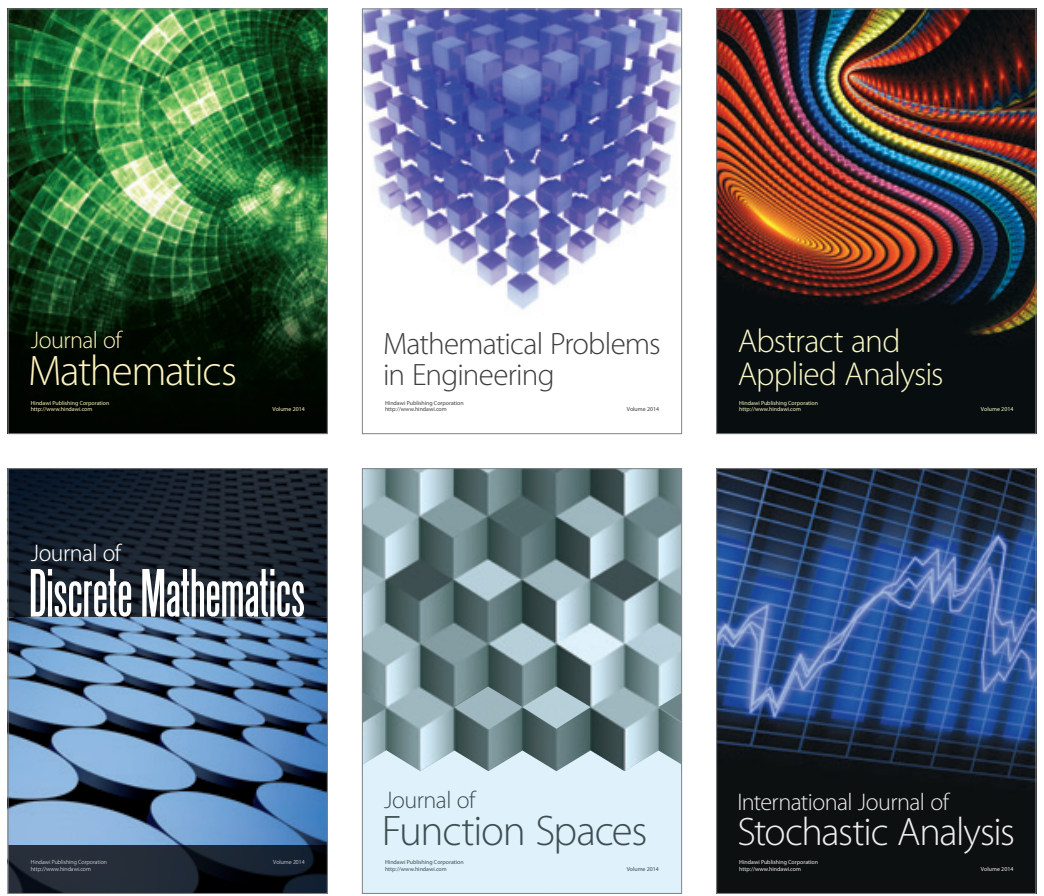

Journal of

Function Spaces

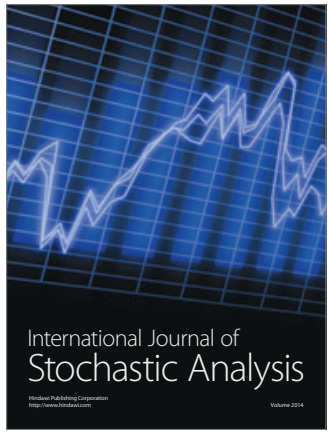

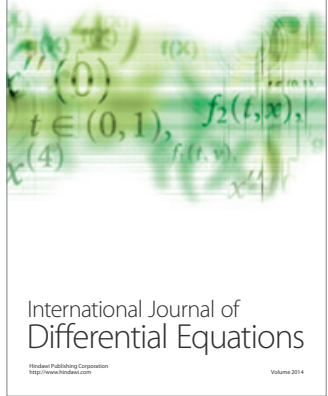
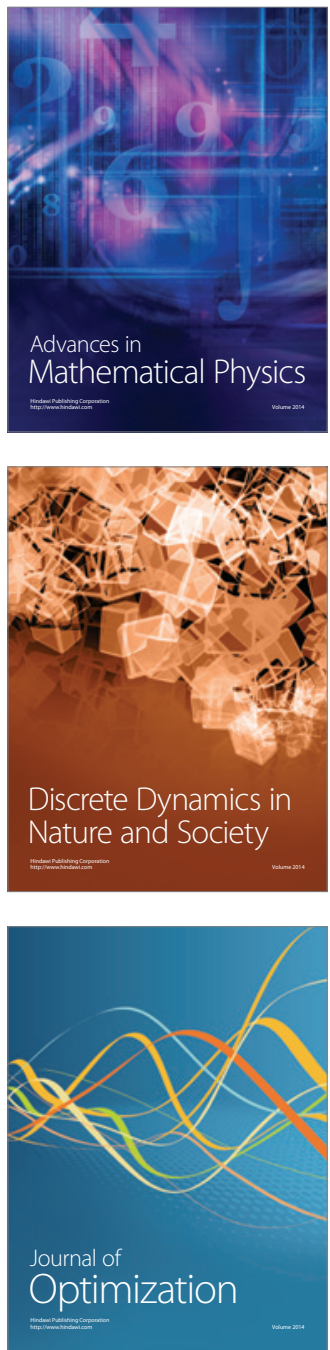